\title{
Classification of acoustic emission signals in wood damage and fracture process based on empirical mode decomposition, discrete wavelet transform methods, and selected features
}

Meilin Zhang, Qinghui Zhang*, Junqiu Li, Jiale Xu and Jiawen Zheng

\begin{abstract}
The nondestructive testing technology of generated acoustic emission (AE) signals for wood is of great significance for the evaluation of internal damages of wood. To achieve more accurate and adaptive evaluation, an AE signals classification method combining the empirical mode decomposition (EMD), discrete wavelet transform (DWT), and linear discriminant analysis (LDA) classifier is proposed. Five features (entropy, crest factor, pulse factor, margin factor, waveform factor) are selected for classification because they are more sensitive to the uncertainty, complexity, and non-linearity of AE signals generated during wood fracture. The three-point bending load damage experiment was implemented on sample wood of beech and Pinus sylvestris to generate original AE signals. Evaluation indexes (precision, accuracy, recall, F1-score) were adopted to assess the classification model. The results show that the ensemble classification accuracies of two tree species reach $94.58 \%$ and $90.58 \%$, respectively. Moreover, compared with the results of the original AE signal, the accuracy of the AE signal processed by the methods proposed is increased by 27.68\%. It indicates that the EMD and DWT signal processing methods and selected features improve the classification accuracy, and this automatic classification model has good AE signal recognition performance.
\end{abstract}

Keywords: Wood, Acoustic emission, Internal damage evaluation, Classification, EMD, DWT, Feature extraction

\section{Introduction}

The wood is a natural composite material with a porous, layered structure. When the wood is locally deformed and fractured, it releases energy in the form of stress waves, which generate a large number of acoustic emission (AE) signals. Although the way of wood damage is very complicated, internal damage can be roughly divided into several basic forms based on microscopic structural change behavior: cell wall buckling and collapse, cell wall interface damage and spallation, formation and extension

*Correspondence: huizq@163.com

School of Big Data and Intelligent Engineering, Southwest Forestry University, Kunming 650224, Yunnan, China of the microcracks damage area, cell wall fracture [1]. Moreover, any type of damage and fracture in the development of its generation will have recognizable features of the AE signal.

As the only active dynamic nondestructive testing method, acoustic emission technology (AET) has been widely used in metal, composite materials, magnetic materials, and other materials defect detection. The beginning of modern AET was in Germany in the early 1950s [2]. The researches on AE in China began in the 1970s, and it was not until the early 1980s that AET began to be applied in engineering practice. Reiterer et al. [3] used the method of combining splitting test and $\mathrm{AE}$ monitoring to study the internal stress change and 
fracture process of cork and hardwood I type fracture. Choi et al. [4] studied the influence of fiber orientation of various composite laminates on $\mathrm{AE}$ characteristics. They divided AE signals into different types through short-time Fourier transform (STFT) and obtained the characteristics of different types of signals. Lamy et al. [5] studied the failure process of Douglas fir under monotonic loading by comparing three experimental methods, force-displacement curve analysis, AE measurement, and digital image acquisition. Fang et al. [6] proposed an improved segmenting cubic Hermite interpolation empirical mode decomposition (EMD) algorithm, which was used for analyzing the characteristics of wood $\mathrm{AE}$ signals, and combined with the instantaneous frequency judgment and AE events statistics. Barile et al. [7] characterized the damage propagation of composite materials by AET. They utilized wavelet packet transform (WPT) to decompose the signal-based data into different levels, and the results could identify the characteristics of different types of damage signals. Li et al. [8] studied the influence of moisture content on AE signals in the process of wood damage, and evaluated the extent of the damage. They used wavelet transform to filter the noise from the original $\mathrm{AE}$ signals, and divided the de-noised $\mathrm{AE}$ signals into deformation AE (DAE) and fracture AE (FAE) signals to the distribution characteristics of frequency. according

Recently, the artificial intelligence method has been applied in the detection of wood damage defects. Jordan et al. [9] modified the initial ultrasonic signal based on the elastic anisotropy of the transmission medium, and then used the neural network system to classify the characteristic signals formed when ultrasonic waves passed through different kinds of wood. Castellani et al. [10] studied the design and training of a multilayer perceptron classifier to identify wood veneer defects based on the statistical features of sub-images of wood. In their study, the evolutionary artificial neural network generation and training (ANNGAT) algorithm was adopted. Compared to manual methods, its complete automation reduces the work of many complex designs. Facciotto et al. [11] used spectrogram analysis techniques to distinguish between different $\mathrm{AE}$ events. And train on different spectral information, which signal sources can be classified and linked to specific emission types with a high level of accuracy. Tha et al. [12] proposed a learning method to detect wood features. It automatically classified defects from wood images using a laser scanner through the deep convolutional neural network (DCNN). The results showed that the overall accuracy of the training model is $99.13 \%$. Wang et al. [13] proposed an AE signal recognition method based on spectrum and acoustic features. This method combined a convolutional neural network $(\mathrm{CNN})$ network with a bidirectional long short term memory (BiLSTM) network to extract features. And then, Softmax was used to realize the recognition of AE signals. Fathi et al. [14] introduced a machine learning-based model to predict the elastic modulus (MOE) and fracture modulus (MOR) of wood with different moisture content using guided wave propagation method. The results showed that the accuracy obtained is higher than that obtained by conventional ultrasonic method. VS et al. [15] provided a fast and nondestructive method for wood identification using a stoichiometric method of Attenuated total reflection Fourier transform infrared (ATR-FTIR) analysis. It used multivariate statistical analysis, including hierarchical cluster analysis (HCA) and principal component analysis (PCA) to identify wood samples.

Most of the above studies used STFT, EMD, WPT, and other processing on $\mathrm{AE}$ signals to identify different damage signal characteristics or implemented a neural network system to train and identify characteristics of different types of wood (spectrum, MOE, MOR, etc.). Because the signals generated in the process of pressurized fracture of wood have the characteristics of uncertainty, complexity, and non-linear, and time-domain characteristic parameters such as signal entropy, crest factor, pulse factor, margin factor, and waveform factor can sensitively reflect the changes of a waveform, so they can be used as a characteristic index to measure the uncertainty of signal state distribution and signal redundancy. Due to its adaptive nature, the EMD algorithm is suitable for processing complex AE signals. Based on the concept of wavelet, the discrete wavelet transform (DWT) discretizes the continuous wavelet. DWT has the characteristics of non-redundant decomposition, and can strictly distinguish the frequency band of AE signals to fully show the time-frequency characteristics of damage, which improves the calculation speed of various data. The linear discriminant analysis (LDA) algorithm is a classic supervised learning algorithm, which can be used not only to reduce the dimension of data, but also as a classifier for predictive analysis. Therefore, in this study, firstly the EMD is employed to process and reconstruct the $\mathrm{AE}$ signals generated during the three-point bending load damage process of wood, and then features are extracted from reconstructed signals with DWT, and finally the LDA method is used to train and test these features, trying to automatically identify or distinguish wood damage and fracture states in different periods based on different acoustic emission characteristics. 


\section{Materials and methods}

Beech and Pinus sylvestris with no defect on the surface and stable moisture content of about $11 \%$ under airdried condition was selected as the experimental material. The sample size was $800 \mathrm{~mm}$ (length) $\times 60 \mathrm{~mm}$ (width) $\times 30 \mathrm{~mm}$ (thickness). The experimental equipment in this study was: (1) NI USB-6366 high-speed acquisition card (National Instruments in US); (2) LabVIEW software to build a 3-channel AE signal acquisition system; (3) UTM5105 universal mechanical testing machine (the maximum test force is $100 \mathrm{kN}$, the power is $1.5 \mathrm{~kW}$, from Shenzhen Sisheng Technology Co., Ltd in China); (4) SR $150 \mathrm{~N}$ single-ended resonant acoustic emission sensor (the signal bandwidth is $25-200 \mathrm{kHz}$, SR $150 \mathrm{~N}$ from Beijing Soundwel Technology Co., Ltd in China); (5) a $40 \mathrm{~dB}$ gain preamplifier (Beijing Soundwel Technology Co., Ltd in China) with a maximum sampling frequency of $2 \mathrm{MHz}$ per channel and an output voltage range of $\pm 5 \mathrm{~V}$.

In this study, three-point bending method was utilized to compress the samples laterally at a velocity of $1 \mathrm{~mm} / \mathrm{min}$ with a span of $200 \mathrm{~mm}$. Three AE sensors are arranged on both sides of the support. In order to avoid interference of signal reception between sensors caused by too close distance, the distance of each two sensors is set as $300 \mathrm{~mm}$. The signal source $\mathrm{P}$ is arranged between $\mathrm{S} 1$ and $\mathrm{S} 2$, and the distance to the two sensors is $150 \mathrm{~mm}$, and the distance to $\mathrm{S} 3$ is $450 \mathrm{~mm}$. The distance between the two supports and source $P$ is $100 \mathrm{~mm}$. The distance between S1/S3 and the nearest end of the sample wood is $100 \mathrm{~mm}$. The experimental structure is illustrated in Fig. 1. And the sensors were coated with silica gel as a coupling agent to ensure more accurate signal acquisition.

Existing studies have shown $[16,17]$ that during the damage process of wood, the frequency of $\mathrm{AE}$ signals can be higher than $300 \mathrm{kHz}$. However, due to the influence of external factors, high-frequency AE signals will be severely attenuated. For example, according to [16, 17], compared with the low-frequency in $60-120 \mathrm{kHz}$, the attenuation of the $\mathrm{AE}$ signal in the frequency range of $270-330 \mathrm{kHz}$ is increased by $20-30 \mathrm{~dB}$. Furthermore, the frequency bandwidth of the sensors used in our study is mainly distributed in the range of $50-200 \mathrm{kHz}$. Therefore, the sampling frequency of AE signals in this study was $500 \mathrm{kHz}$. According to Shannon's sampling theorem, AE signals in the range of $0-250 \mathrm{kHz}$ can be theoretically identified. First, the sensors receive AE signals caused by the pressure on the sample surface and perform the sound-electricity transformation. Then a preamp is used to amplify the transformed signals. Finally, the data acquisition card converts the amplified signal to digital signals, and stores them in a computer. The process is illustrated in Fig. 2. The signals of each sensor were stored in a text file for subsequent research.

AE signals can be used to assess the internal fracture state of the sample wood. However, the assessment directly based on the amplitude or energy of AE signals is susceptible to factors such as amplification and noise of the acquisition system, and cannot be adaptive. Thus, for a more accurate and adaptive evaluation for the wood damage, the original signal should is processed and analyzed, and trying to find features with higher sensitivity and robustness. In this study, the signal analysis workflow is shown in Fig. 3.

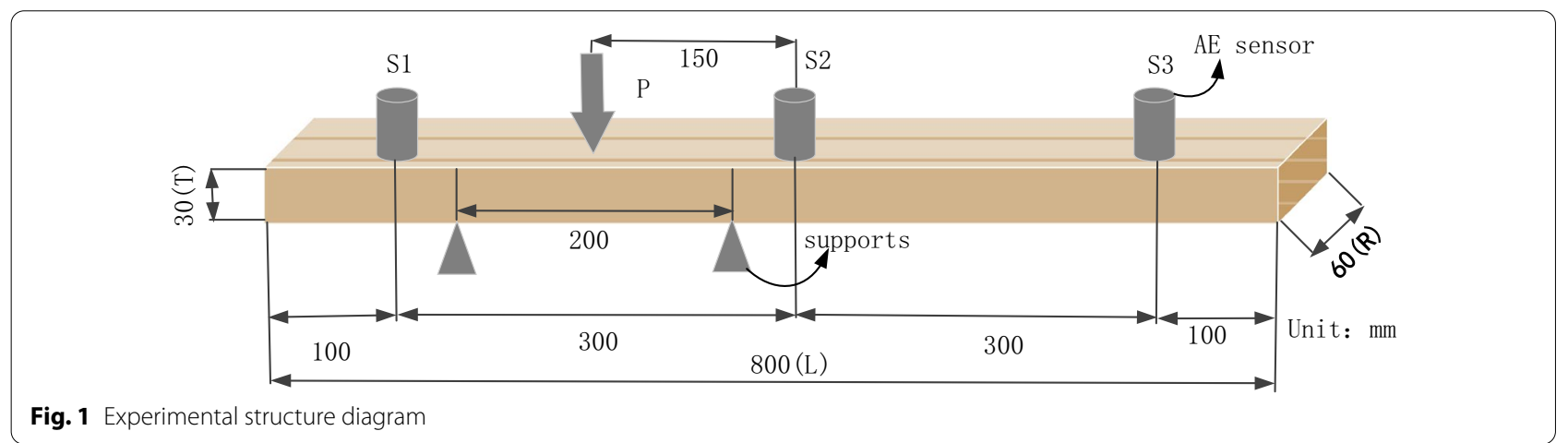

Fig. 1 Experimental structure diagram

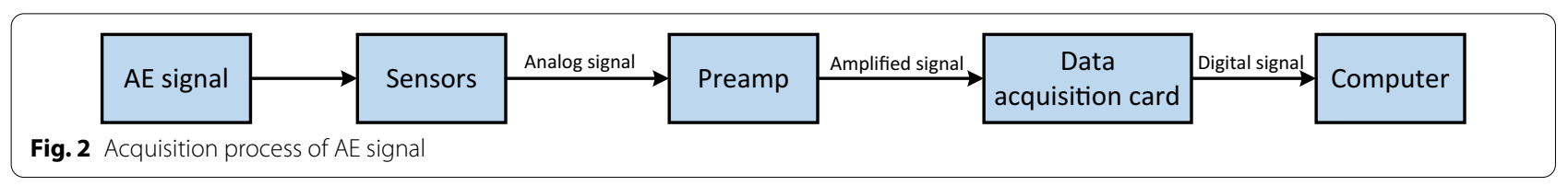




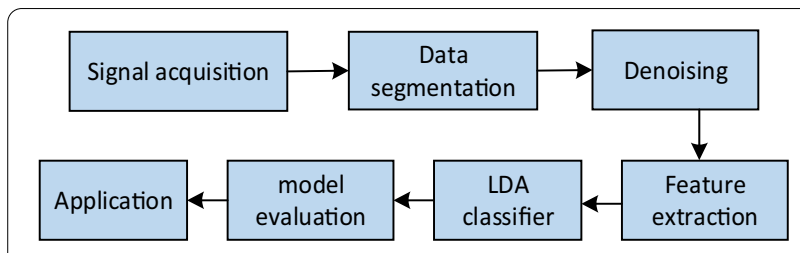

Fig. 3 The processing of AE signals

The hardware and software experimental environment of signal processing is listed in Table 1.

\section{Signal preprocess based on EMD EMD algorithm basics}

Due to influences of the external environment, signals collected contain much noise. In this study, the empirical mode decomposition (EMD) method is utilized to filter interference from original signals. The EMD was first proposed by the American scholar Huang [18] in 1998. It is based on the local characteristics of the signal in the time-domain, which is adaptive and suitable for analyzing and processing non-stationary and non-linear complex signals. It can clearly distinguish the eigenmodes of overlapping complex data sequences, filter out the interference as needed, and extract informative signals.

Its basic principle is to decompose a discrete data signal into a collection of intrinsic mode functions (IMF) with different frequencies. Thus, the original signal $X(t)$ can be expressed as the sum of IMFs and a residue, as shown in Eq. (1):

$$
X=\sum_{i=0}^{n} c_{i}+r
$$

where $c_{i}$ is IMF, $r$ is residue.

Then, some of the IMFs can are retained or removed and reconstructing the signals based on a specific mechanism. Thus, the valuable information was preserved while removing the interference.

\section{Signal reconstruction mechanism}

As mentioned, the frequencies of the AE signal collected in this study are mainly distributed in the range of
50-200 kHz. Moreover, AE signals have greater energy and more impact components. Therefore, in this study, we comprehensively considered the energy and instantaneous frequency of each signal component, as well as the kurtosis, and used them as the basis for signal reconstruction.

In signal processing, the instantaneous frequency is one of the most important features of a signal. It is a monotone function of time, and at a given moment, a signal only has a unique instantaneous frequency. That restricts the signal in a "narrowband". Intuitively, the instantaneous frequency is the differential of the phase. In addition, the kurtosis reflects the numerical statistics of the waveform distribution characteristics. When there are more impact components in a signal, its kurtosis value increases obviously. When subjected to external forces, a wood's inner wall changes while it releases energy in the form of stress waves, namely AE signals. Obviously, these $\mathrm{AE}$ signals contain more impact components. Furthermore, it can be believed that among the IMFs obtained by EMD, some of the IMFs with more impact components have more useful information. Correspondingly, the kurtosis values of these IMFs signals will be greater than that of others. Thus, selecting IMFs based on their kurtosis will help to more effectively extract useful information from the original signals. After a segment of the signal is decomposed by EMD, the kurtosis value of each IMF can be calculated according to Eq. (2) [19], where $T_{i}$ is the $i$ th IMF component kurtosis:

$$
T_{i}=\frac{1}{n} \sum_{k=1}^{n} c_{i k}^{4} .
$$

\section{Feature extraction and dimension reduction}

In most classification algorithms, the computational cost is related to the size or dimension of the data sample. Thus, it is necessary to perform feature extraction on signals to reduce their size or dimension while retaining valuable information. Feature extraction will not only reduce the complexity of computation but also improves the robustness of classification algorithms. In this study, except for the time-frequency features extracted by the

\begin{tabular}{|c|c|c|c|}
\hline \multicolumn{2}{|c|}{ Hardware environment } & \multicolumn{2}{|c|}{ Software environment } \\
\hline Memory & $16 \mathrm{~GB}$ & System & Windows 10 \\
\hline $\mathrm{CPU}$ & $\begin{array}{l}\text { Intel (R) Core (TM) i5-10210U CPU @ } 1.60 \text { GHz (8 } \\
\text { CPUs), 2.1 GHz }\end{array}$ & Environment & Tensorflow-gpu 2.4.1 \\
\hline DxDiag Version & 10.00.19041.0928 64 bit Unicode & configuration & VS2019+ Python 3.8 + keras 2.4 .3 \\
\hline
\end{tabular}

Table 1 Experimental environment 
DWT algorithm, entropy and some statistical features related to the shape of the waveform also are extracted.

\section{Discrete wavelet transform}

Wavelet provides time-scale information of signals and can extract time-varying features, which makes wavelet transform (WT) an ideal tool for analyzing transient or non-stationary signals [20]. Continuous wavelet transform (CWT) can be expressed as Eq. (6):

$$
\operatorname{CWT}_{(a, b)}=\frac{1}{\sqrt{|a|}} \int f(t) \varphi \times\left(\frac{t-b}{a}\right) \mathrm{d} t,
$$

where $\varphi(t)$ is the mother wavelet; $a$ represents the scale coefficient, and it is the reciprocal of frequency; $b$ represents the shift coefficient. The DWT is derived from the discretization of CWT $(a, b)$. It can not only avoid a large amount of information redundancy caused by continuous changes in scale and displacement, but also greatly save computation time while providing sufficient information. It is a very effective time-frequency localization analysis method. It is calculated by:

$$
\operatorname{DWT}_{(a, b)}=\frac{1}{\sqrt{2^{j}}} \int f(t) \varphi \times\left(\frac{t-2^{j k}}{2^{j}}\right) \mathrm{d} t,
$$

where $a$ and $b$ are replaced by $2^{j}$ and $2^{j k}$. The DWT can accurately describe the signal features by the wavelet basis functions of different scale transformations, and constantly focus on any small details of the signal.

\section{Entropy}

Entropy represents the overall information uncertainty of random objects in the sense of average, which is a very important concept in information theory. For a discrete random variable $X$, its probability space is set as [21]:

$$
\left[\begin{array}{l}
X \\
P(x)
\end{array}\right]=\left[\begin{array}{l}
a_{1}, a_{2}, \ldots, a_{n} \\
p\left(a_{1}\right), p\left(a_{2}\right), \ldots, p\left(a_{n}\right)
\end{array}\right],
$$

where the probability $p$ is:

$$
0 \leq p\left(a_{i}\right) \leq 1, \sum_{i=1}^{n} p\left(a_{i}\right)=1 .
$$

Then the average uncertainty of the whole probability space, namely information entropy, is defined as:

$$
H(x)=-\sum_{i=1}^{q} p\left(a_{i}\right) \log p\left(a_{i}\right) .
$$

Information entropy is employed to describe the redundancy of information sources. The more ordered a signal is, the lower the information entropy is. Conversely, the more chaotic a signal is, the higher the entropy of information is.

\section{Other statistical characteristics}

Some time-domain characteristic parameters such as crest factor, pulse factor, margin factor, and waveform factor can sensitively reflect the change of a waveform, and are often used as characteristic parameters of abnormal waveform detection. Let a discrete signal sequence $X=\left(x_{1}, x_{2} \ldots x_{\mathrm{n}}\right), n$ is the signal length, and the calculation of each digital characteristic parameter is as follows [22].

The crest factor is the ratio of the signal peak difference $\left(X_{\text {peak }}\right)$ to the root mean square value $\left(X_{\text {rms }}\right)$, representing how extreme the peaks are in a waveform. As Eq. (7):

$$
X_{C}=\frac{X_{\mathrm{peak}}}{X_{\mathrm{rms}}}=\frac{X_{\mathrm{max}}-X_{\mathrm{min}}}{\sqrt{\frac{1}{n} \sum_{i=1}^{n} x_{i}^{2}}} .
$$

The impulse factor is the ratio of the signal peak difference $\left(X_{\text {peak }}\right)$ to the average absolute value $\left(X_{\text {arv }}\right)$. As Eq. (8)

$$
X_{I}=\frac{X_{\text {peak }}}{X_{\text {arv }}}=\frac{X_{\max }-X_{\min }}{\frac{1}{n} \sum_{i=1}^{n}\left|x_{i}\right|} .
$$

The margin factor is the ratio of the signal peak difference $\left(X_{\text {peak }}\right)$ to the root amplitude $\left(X_{\mathrm{r}}\right)$. The root mean square is the arithmetic square root of the mean value of the sum of the squares of the signals, and the root amplitude is the square of the mean value of the arithmetic square roots. As Eq. (9):

$$
X_{L}=\frac{X_{\text {peak }}}{X_{r}}=\frac{X_{\max }-X_{\min }}{\left(\frac{1}{n} \sum_{i=1}^{n} \sqrt{|x|}\right)^{2}} .
$$

The waveform factor is the ratio of the root mean square value $\left(X_{\text {rms }}\right)$ to the rectification mean value $\left(X_{\text {arv }}\right)$, namely the ratio of the pulse factor to the crest factor. As Eq. (10):

$$
X_{S}=\frac{X_{\mathrm{rms}}}{X_{\mathrm{arv}}}=\frac{\sqrt{\frac{1}{n} \sum_{i=1}^{n} x_{i}^{2}}}{\frac{1}{n} \sum_{i=1}^{n}\left|x_{i}\right|} .
$$

\section{Method of classification Linear discriminant analysis}

Linear discriminant analysis (LDA) [23] is widely used in statistics, machine learning, and pattern recognition. It is based on Fisher's criterion, which is a generalization of Fisher's linear discriminant (FLD) method. As a 
supervised learning method, it can be not only used to reduce the dimension of data, but also used as a classifier for predictive analysis. The principle of LDA is to find the optimal projection matrix. Its process is to carry out low-dimensional projection on a set of linear and divisible data to make the distance between data of different classes as far as possible and the distance between similar data as small as possible, to achieve the standards of dispersion degree of minimum intra-class and maximum inter-class. Then the projection matrix is used for dimensionality reduction of the original data to obtain a low-dimensional sample set with good separability for subsequent classification modeling and prediction. Closely related to the analysis of variance and regression, LDA aims to represent other measurements as dependent variables or linear combinations. The difference between them is that the former uses numerical quantities as dependent variables, while LDA uses classification variables.

\section{Evaluation indicators}

Indicators to evaluate the quality of the model include accuracy, precision, recall, F1-score, etc. But these indicators are usually applicable to binary classification problems, so this paper defines precision, accuracy, recall, and F1-score applicable to multiple classifications. Assuming there are $n(n>2)$ classes of data, then the values of precision $(P)$, accuracy $(A)$, recall $(R)$, and F1-score (F1) of multiple classifications are defined as follows [20]:

$$
\begin{aligned}
& P=\frac{\sum_{i=1}^{n} \mathrm{TP}_{\mathrm{i}}}{\sum_{i=1}^{n}\left(\mathrm{TP}_{i}+\mathrm{FP}_{\mathrm{i}}\right)}, \\
& A=\frac{\sum_{i=1}^{n}\left(\mathrm{TP}_{i}+\mathrm{TN}_{i}\right)}{\sum_{i=1}^{n}\left(\mathrm{TP}_{i}+\mathrm{FP}_{i}+\mathrm{TN}_{i}+\mathrm{FN}_{i}\right)}, \\
& R=\frac{\sum_{i=1}^{n} \mathrm{TP}_{i}}{\sum_{i=1}^{n}\left(\mathrm{TP}_{i}+\mathrm{FN}_{i}\right)} \\
& F 1=\frac{2 \times P \times R}{P+R},
\end{aligned}
$$

where $i=1,2 \ldots, n$ is the total number of sample categories. TP is true positive, $\mathrm{FP}$ is false positive, $\mathrm{FN}$ is true negative, and TN is false negative, as shown in Table2, that is, TP: it is predicted to be positive, and it is actually
Table 2 TP, FP, FN, TN

\begin{tabular}{lll}
\hline & Labeled as positive & Labeled as negative \\
\hline Predicted as positive & True positive (TP) & False positive (FP) \\
Predicted as negative & False negative (FN) & True negative (TN) \\
\hline
\end{tabular}

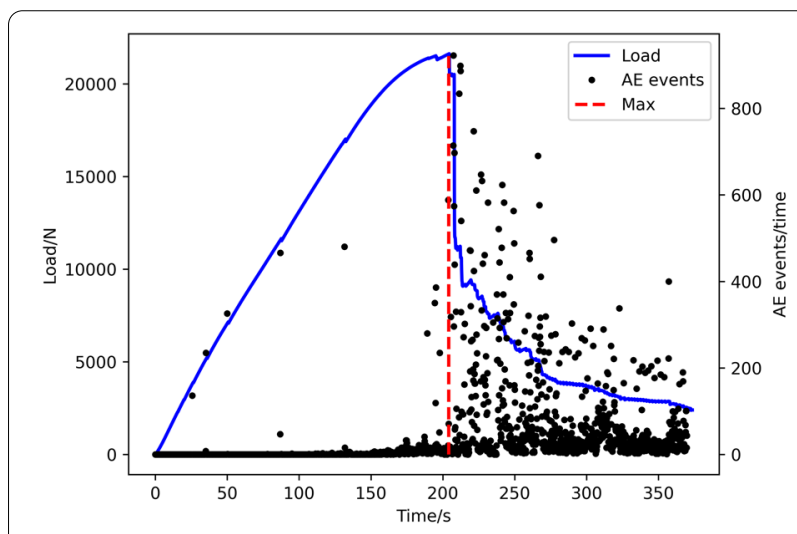

Fig. 4 Time-load curve

positive; TN: it is predicted to be negative, and it is actually negative; FP: it is predicted to be positive, while it is actually negative; FN: it is predicted to be negative, while it is actually positive.

\section{Results and analysis \\ Beech \\ Original signal obtained from experiment}

The duration of this experiment was 370s, and the sampling frequency was $500 \mathrm{kHz}$. In order to analyze the relationship between stress and $\mathrm{AE}$ signal generation, the corresponding time-load curve and the scatter distribution of $\mathrm{AE}$ events were drawn (Fig. 4). Two milliseconds was taken as a temporal window, and the threshold method was employed to calculate the number of $\mathrm{AE}$ events. The time-load curve shows that the load curve increases uniformly with the increasing stress and reaches a maximum value at about 204th second. Before this time, the number of $\mathrm{AE}$ events increased sharply only in a few time periods. After this time, the number of $\mathrm{AE}$ events has increased dramatically, and the load curve decreases sharply, indicating that the sample wood fractures at this moment. Then, the curve decreases gradually at a slower rate, and the number of $\mathrm{AE}$ events also decreases along with it. By observing the curve and the $\mathrm{AE}$ event scatter plot, it can be seen that the load curve or the number of $\mathrm{AE}$ events has a certain correlation, and both can reflect the internal damage state of the wood to a certain extent. However, they cannot accurately demonstrate the various stages of internal damage to the wood. 
The original AE signal of the test process is illustrated in Fig. 5, which contains $1.85 \times 10^{8}$ data points according to the sampling frequency and duration. From Fig. 5 and the distribution of AE events in Fig. 4, it can be easily observed that many $\mathrm{AE}$ signals have been generated before the load reaches the maximum, which indicates that microcracks have been inside the sample wood. As mentioned before, internal damages of wood can be roughly divided into four basic types from the microscopic point of view. Thus, in this study, the original signal was also divided into four segments according to its amplitude, corresponding to these four types. $0-100 \mathrm{~s}$ can be regarded as a small amount of AE signal caused by wood cell wall buckling and collapse, which is called buckling AE signal. 100-150 s can be regarded as a stable small amplitude AE signal caused by the damage and delamination of wood cell wall interface, which is called deformation $\mathrm{AE}$ signal. 150-200 s can be regarded as $\mathrm{AE}$ signals that are more chaotic and high-energy than those in the second stage due to the formation and expansion of wood micro-crack damage zone, which is called micro-crack AE signal. 200-370 s produced a large number of long lasting and complex AE signals, which can be considered as AE signals caused by wood cell wall fracture, and this $\mathrm{AE}$ signal is called fracture $\mathrm{AE}$ signal.

\section{Signal decomposition and reconstruction}

Figure 6 shows the time-domain signals corresponding to each IMF component after EMD decomposition of $5 \times 10^{4}$ sample data. It can be observed that the signal energy is mainly concentrated in the first five IMF components. Figure 7 shows the kurtosis and the mean value of instantaneous frequency of IMF components. It can be learned that the normalized kurtosis values of the first two IMFs are much greater than that of other IMFs, and the IMFs with the greater kurtosis values contain more useful information. Moreover, Fig. $7 \mathrm{~b}$ shows that the power of $\mathrm{AE}$ signals is mainly concentrated in the first

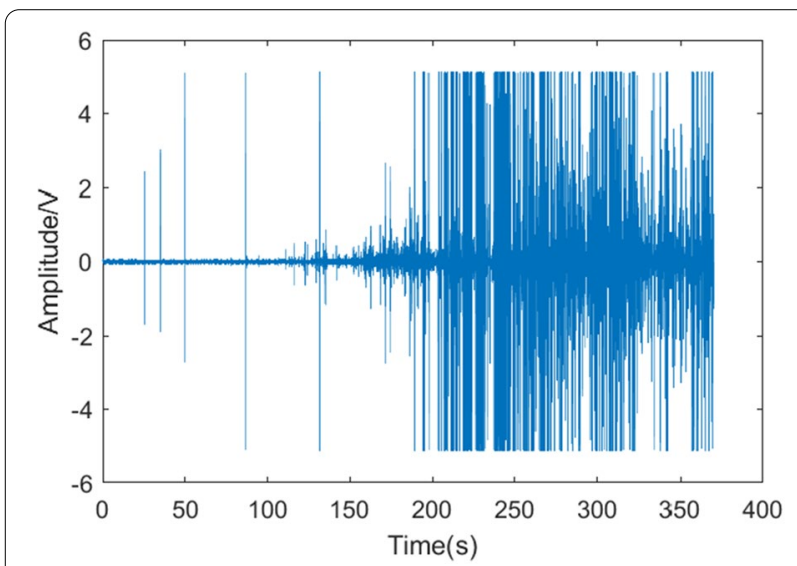

Fig. 5 Original acoustic emission signal four IMFs. Therefore, considering signal energy, kurtosis, and instantaneous frequency comprehensively, the first four IMFs were selected to reconstruct AE signals.

\section{Dataset and segmentation}

In this study, DWT was used to extract the frequency domain features of AE signals. Due to its good smoothing effect, the $\mathrm{db} 4$ wavelet was selected as the basis function. The reconstructed signals were decomposed by an 8-layer discrete wavelet. The detailed components of the eight scales $\mathrm{CD} 1-\mathrm{cD} 8$ and the approximate component of the eighth scale cA8 were selected as the frequency domain features of AE signals. Figure 8 shows the timefrequency signals obtained by DWT decomposition of $10^{4}$ sample data. It can be seen that the length of data for each approximate component from $\mathrm{cD} 1-\mathrm{cD} 8$ decreases layer by layer, thus greatly reducing the subsequent computation time for feature extraction.

For better computational efficiency, 10,000 data points are treated as a sub-segment, corresponding to a period of $0.02 \mathrm{~s}$, and used it as the basic unit for subsequent processing. Thus, there were 18,500 sub-segments in this study, and the number of sub-segment in each type is determined by the total amount of data in this type. Then, the DWT decomposition was performed for each sub-segment separately and calculating feature values (entropy, crest factor, impulse factor, margin factor, and waveform factor) for eight layers of detail components, one layer approximate component, and the mean ratio of absolute values in each layer. Finally, a dataset was obtained, containing $18,500 \times 53(5 \times 9+8)$ feature data.

Moreover, it should be pointed out that the length of the sub-segment may influence the value of the extracted features, thereby affecting the classification result. However, the focus of this article is on the characteristics and processing methods of $\mathrm{AE}$ signals, rather than data length. Furthermore, for $\mathrm{AE}$ signals with a frequency range of $50-200 \mathrm{kHz}$, a sub-segment with a duration of $0.02 \mathrm{~s}$, selected in our study, almost cover all potential information.

\section{Training and test results based on LDA}

The LDA was used to conduct training and testing on obtained dataset (70\% for training, 30\% for validation). Figure 9 is the confusion matrix obtained. The evaluation indexes (precision, recall, F1-score, micro-average, macro-average, and weighted average) were calculated according to the confusion matrix, and the results are shown in Table 3. Overall, this model achieves a high classification performance. As shown, the evaluation indexes of the four types all almost reach 0.95 .

Figure 10 shows the curves of model training accuracy and loss value. In general, the larger the area (AUC) 


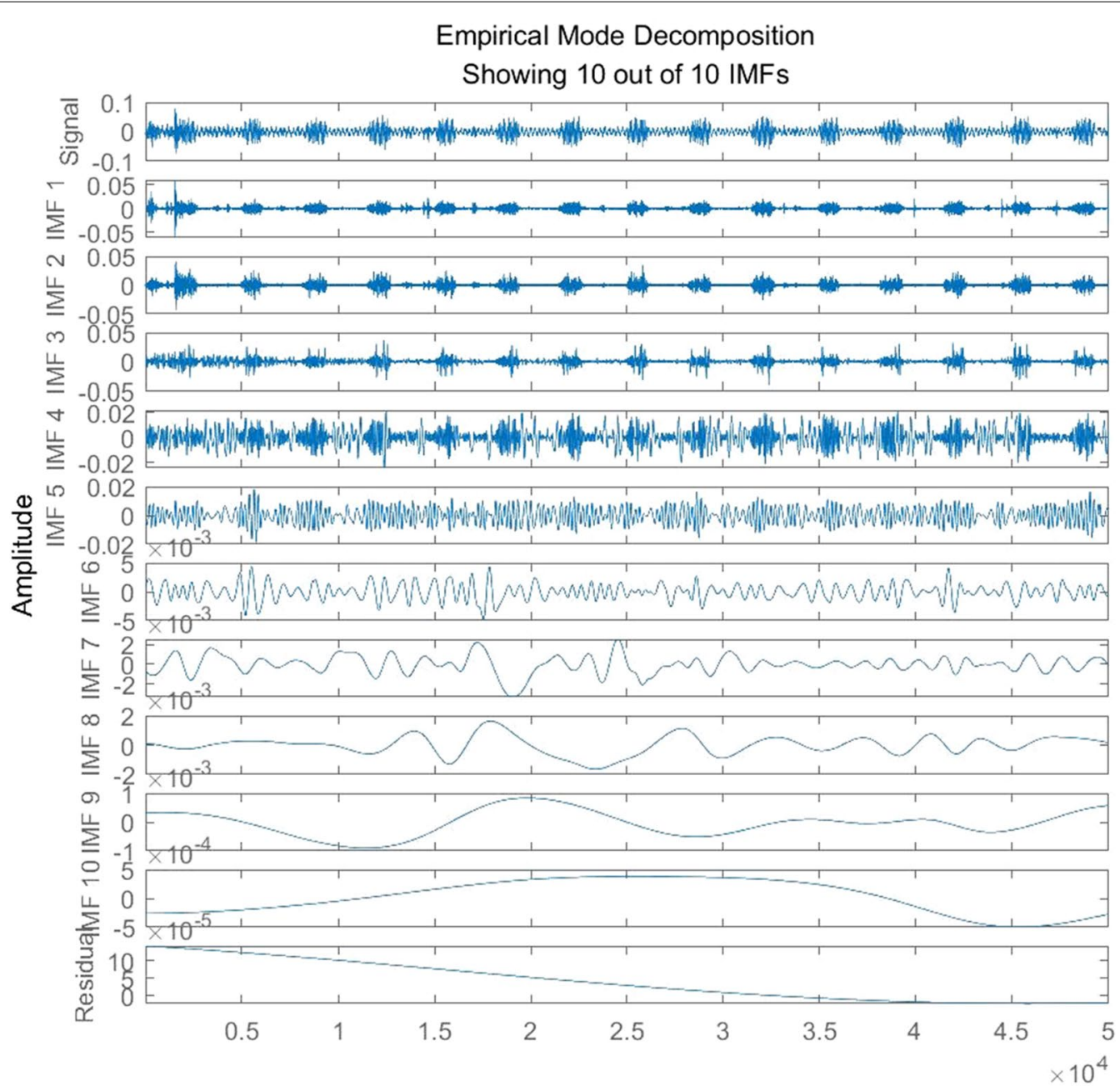

Fig. 6 EMD decomposition of AE signals

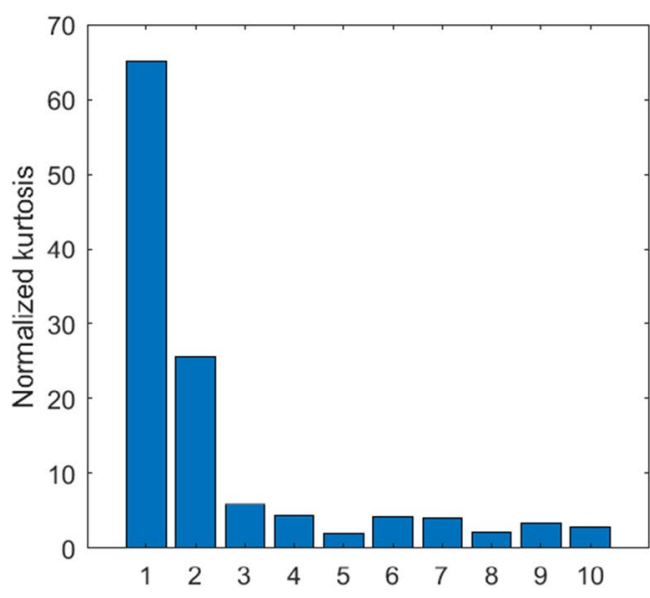

(a)IMF component

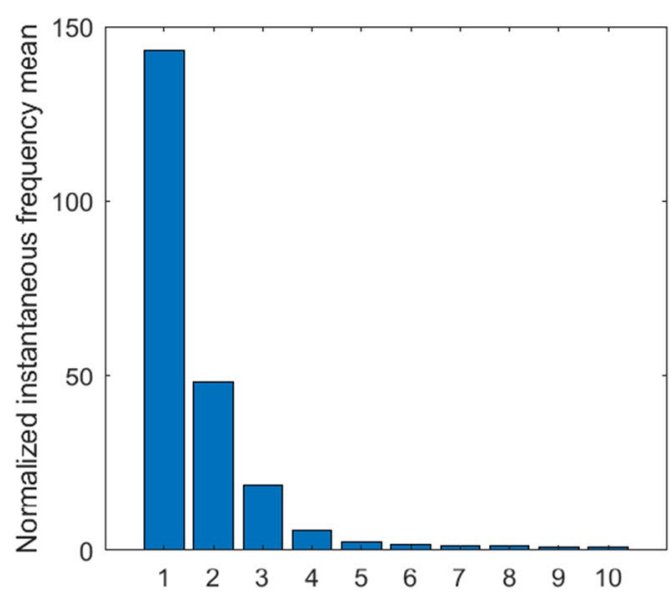

(b)IMF component

Fig. 7 Normalized kurtosis/instantaneous frequency 

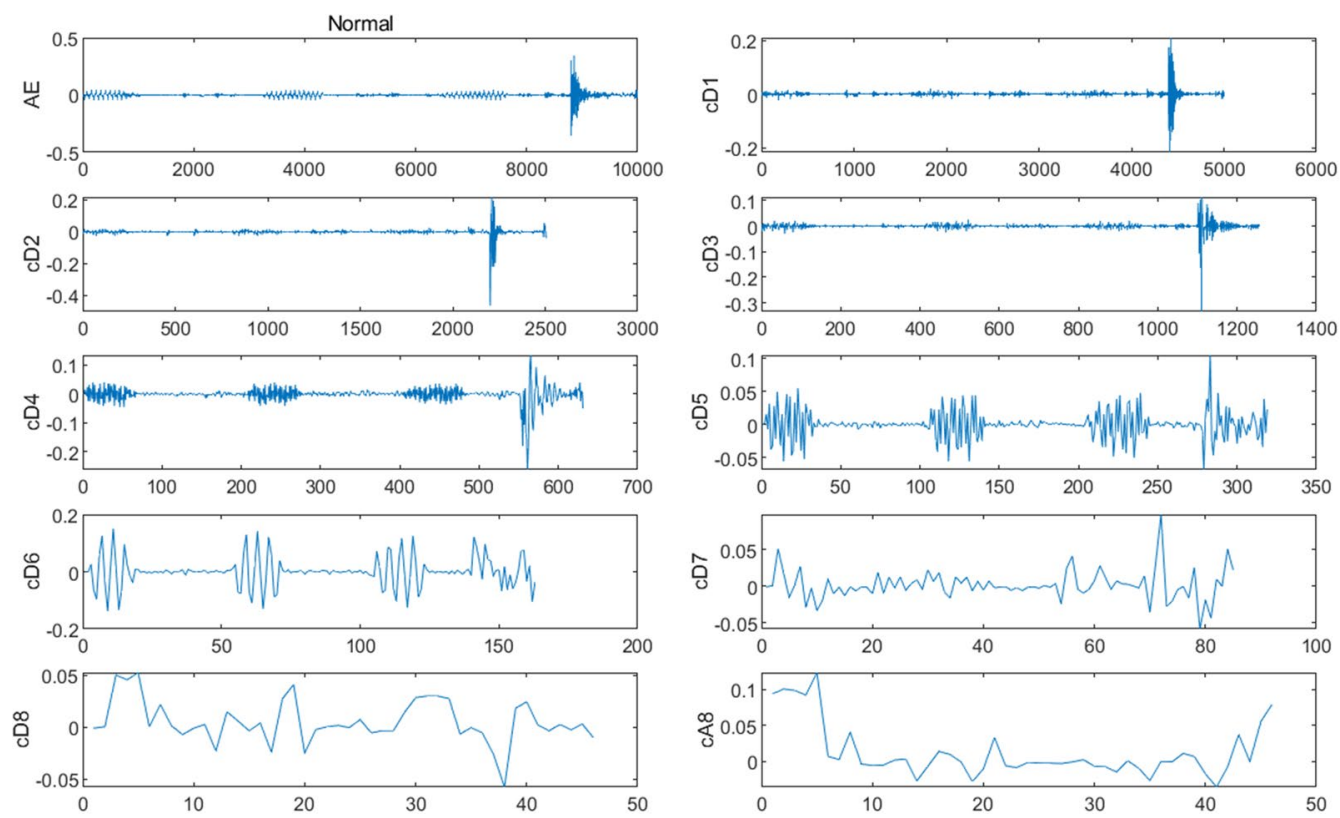

Fig. 8 Discrete wavelet transform of AE signal

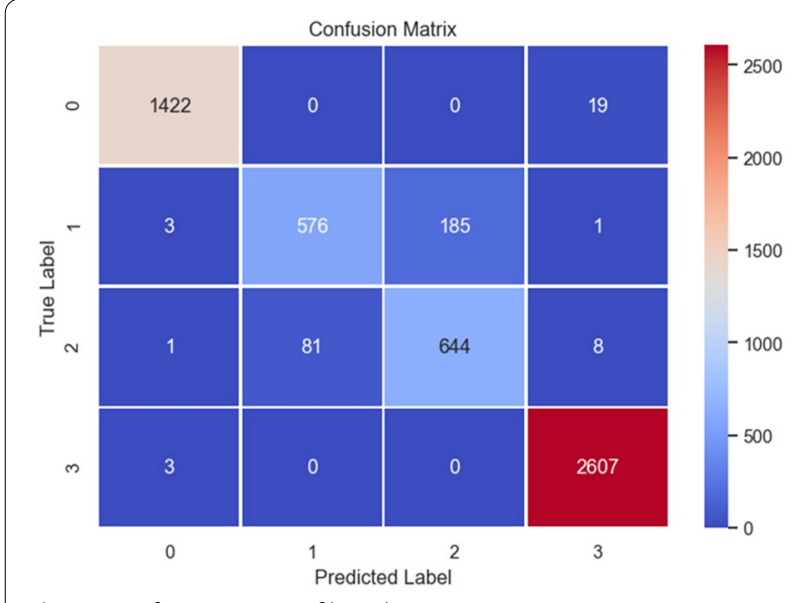

Fig. 9 Confusion matrix of beech

under the accuracy (ACC) curve is, or the closer the curve is to the upper left corner, the more accurate the training model will be. From Fig. 10, it can be observed that as the number of training iterations increases gradually, the total loss value shows an overall trend of decline and converges after a certain number of iteration steps. This demonstrates that the model is of good convergence characteristics. Furthermore, the relations of the training curve and the validation curve in Fig. 10 indicate that the model did not overfit or underfit. Moreover, it can be noticed that from those figures and table the classification of the Type 0 and Type 3 is very accurate, while that
Table 3 Evaluation indicators

\begin{tabular}{lllll}
\hline \multicolumn{2}{l}{ Classification report } & & & \\
\hline & Precision & Recall & F1-score & Support \\
\hline 0 & 1.00 & 0.99 & 0.99 & 1441 \\
1 & 0.88 & 0.75 & 0.81 & 765 \\
2 & 0.78 & 0.88 & 0.82 & 734 \\
3 & 0.99 & 1.00 & 0.99 & 2610 \\
Micro-avg & 0.95 & 0.95 & 0.94 & 5550 \\
Macro-avg & 0.91 & 0.90 & 0.90 & 5550 \\
Weighted avg & 0.95 & 0.95 & 0.95 & 5550 \\
\hline
\end{tabular}

of the Type 1 and the Type 2 has a higher misjudgment rate. For example, it can be learnt from the confusion matrix that 185 data of the Type 1 are misclassified as the Type 2, while 81 data of the Type 2 are misclassified as the Type 1 . In fact, the AE signals change gradually with the increase of pressure, and there is no strict boundary between the Type 1 and Type 2 of AE signals. In other words, the two types of AE signals, especially those that are close to the designated classification boundary, have high similarity. In addition, the data numbers of these two types are less than that of the other two types. Consequently, it is reasonable for a certain number of misjudgments to occur.

Furthermore, the purpose of our classification is to automatically detect and identify the internal damage of the wood, so even if there are a certain number of misjudgments, it will not affect our ultimate goal. The reason 
is that in the process of detection and identification, if the $\mathrm{AE}$ signals are frequently and alternately recognized as the second or third type, it indicates that the wood is in the transitional stage of deformation and microcracking.

\section{Comparative analysis}

To illustrate the performance of the signal processing methods introduced in this study, the classification results of unprocessed and processed signals are compared, as shown in Table 4. From Table 4, it can be learned that the classification performance of unprocessed signals is lower than that of others. Its accuracy can only reach about $75 \%$. However, no matter which signal processing method is adopted, the classification performance can be improved to a certain extent. For example, compared with the results of unprocessed $\mathrm{AE}$ signal, the overall accuracy of the AE signal processed by EMD and DWT is increased by $27.68 \%$. Thus, it can be concluded that the signal processing methods proposed in this study do improve classification accuracy.

Furthermore, a comparative study is conducted on the influence of different features on the classification results. Here, three feature groups are used: Type 1 included some common statistical features, such as mean, standard deviation, skewness, median, etc. Type 2 contained features introduced in this study. Type 3 took all features mentioned before. The classification results are shown in Table 5. As shown, the accuracy, precision, recall, and F1-score of Type 1 are $0.820,0.809,0.820$, and 0.806 , respectively. Those of Type 2 are $0.946,0.947,0.946$, and 0.945 , respectively, and the overall accuracy is increased by $16.26 \%$. However, the classification result of Type $1+2$ is slightly lower than that of Type 2 . It indicates that more features do not mean higher accuracy. The reason is that the existence of redundant features with reduced discriminative power may confuse the classifier. Thus the

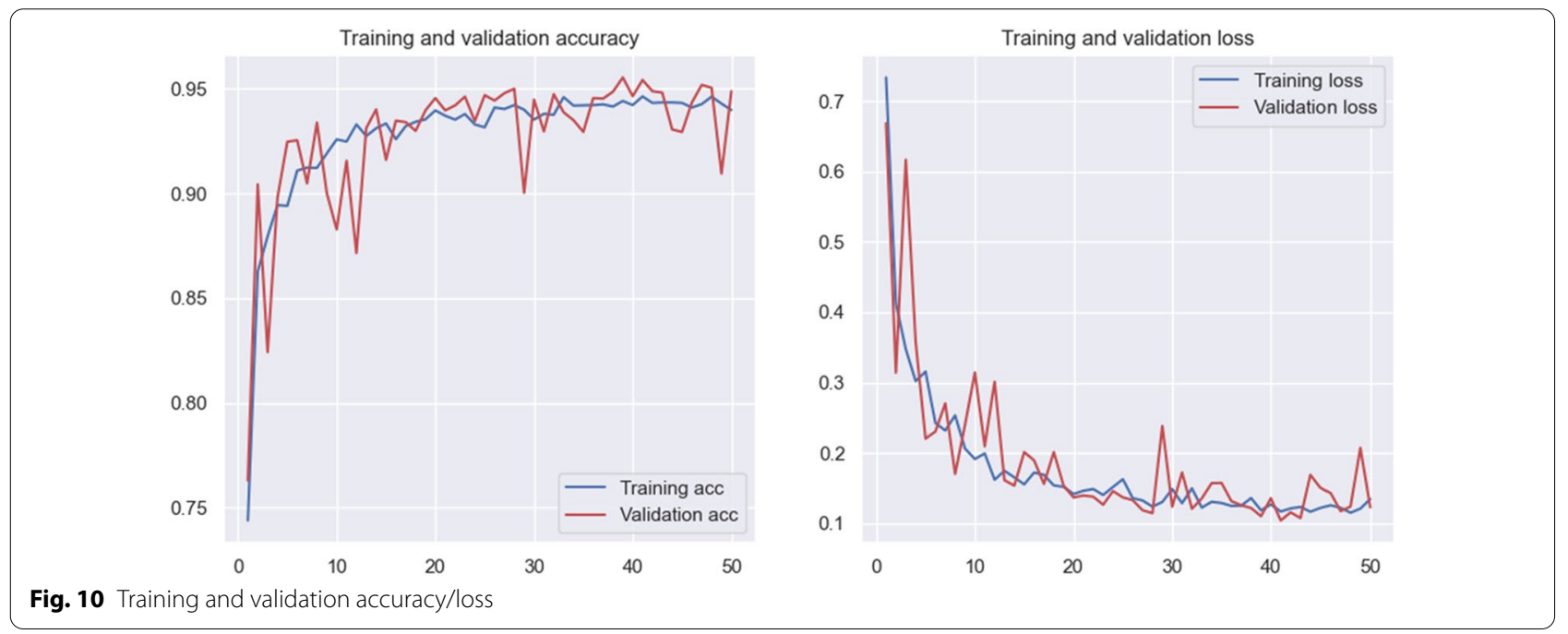

Table 4 Comparison of classification results between unprocessed signals and processed signals

\begin{tabular}{lllll}
\hline & Accuracy & Precision & Recall & F1-score \\
\hline Unprocessed signals & 0.748 & 0.740 & 0.748 & 0.728 \\
EMD & $0.779(4.14 \%)$ & $0.791(6.89 \%)$ & $0.779(4.14 \%)$ & $0.766(5.22 \%)$ \\
DWT & $0.847(13.24 \%)$ & $0.837(13.11 \%)$ & $0.847(13.24 \%)$ & $0.832(14.29 \%)$ \\
EMD+DWT & $0.946(26.47 \%)$ & $0.947(27.97 \%)$ & $0.946(26.47 \%)$ & $0.945(29.81 \%)$ \\
\hline
\end{tabular}

Table 5 Comparison of classification results between different feature types

\begin{tabular}{lllll}
\hline & Accuracy & Precision & Recall & F1-score \\
\hline Feature Type 1 & 0.820 & 0.809 & 0.820 & 0.806 \\
Feature Type 2 & $0.946(15.37 \%)$ & $0.947(17.06 \%)$ & $0.946(15.37 \%)$ & $0.945(17.25 \%)$ \\
Feature Type 1+2 & $0.921(12.32 \%)$ & $0.924(14.22 \%)$ & $0.921(12.32 \%)$ & $0.920(14.14 \%)$ \\
\hline
\end{tabular}


feature selection is of ultimate significance in producing a more accurate model. Even so, it is not the focus of this article. And the results demonstrate that entropy and other features related to signal shape are more useful in AE signal detection and classification.

\section{Pinus sylvestris}

To further verify the method proposed in this article, the same three-point bending experiment was utilized on the sample wood of Pinus sylvestris, and AE signals were collected and processed with the same method. All the experiment settings are the same as that of the previous test. The results are as follows.

\section{Original signal obtained from experiment}

The test lasted $281 \mathrm{~s}$ and the sampling frequency was $500 \mathrm{kHz}$, so $1.405 \times 10^{8}$ data signals were obtained. The original AE signals are shown in Fig. 11. In order to analyze the relationship between stress and $\mathrm{AE}$ signal generation, the same experimental method as the $\mathrm{AE}$ experiment of beech was adopted, and the corresponding time-load curve and the scatter distribution of $\mathrm{AE}$ events was plotted (Fig. 12). It can be seen from Fig. 11 that there is almost no AE signal before the 50th second, and some AE signals are generated between $50-80 \mathrm{~s}$, and the amplitude gradually increases. After the 80th second, a large number of AE signals are generated. And most of them can reach the maximum amplitude. At the same time, it can be seen from Fig. 12 that the load reaches its maximum value at the 216th second, and a large number of $\mathrm{AE}$ events have occurred before this time. After this time, the number of acoustic emission events has increased significantly.

It should be noted that, unlike that of beech, the amplitude of the AE signals of Pinus sylvestris reached the maximum at 80th second prefer to at the moment that the load reached the maximum. The difference in the

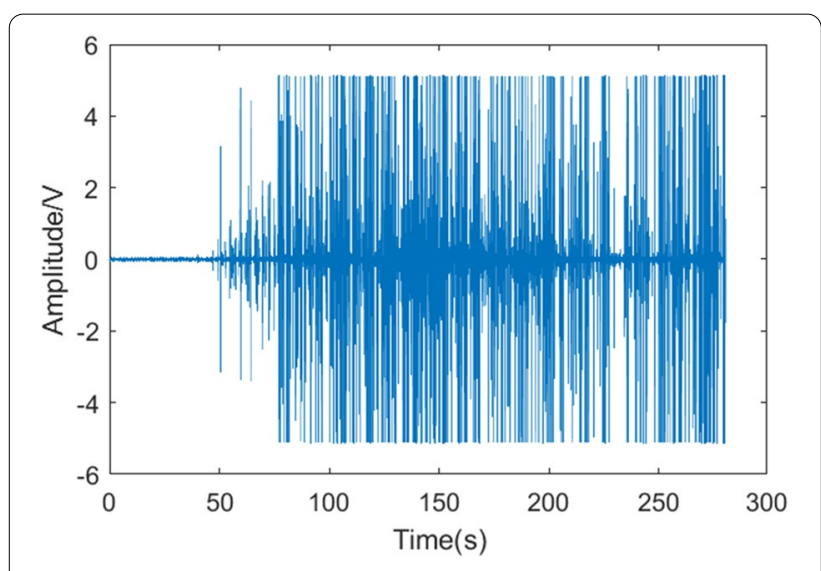

Fig. 11 Original acoustic emission signal of Pinus sylvestris

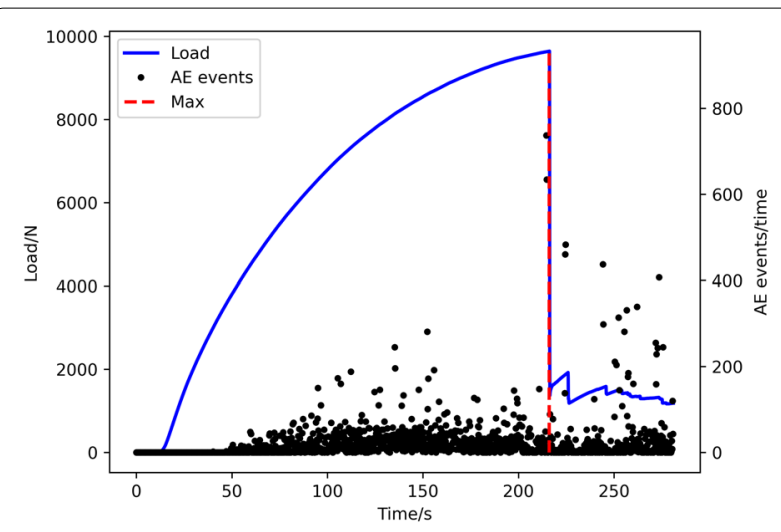

Fig. 12 Time-load curve

type or internal structure of the wood samples leads to this result. However, this also persuasively demonstrates why it is difficult to accurately judge the internal damage state of the sample wood only by the amplitude of the AE signal. In addition, it can be seen from Fig. 12 that the number of $\mathrm{AE}$ times in different time periods does not have a clear dividing line. Therefore, it is also difficult to classify AE signals by the number of AE events.

\section{Test results based on LDA classification model}

In order to accurately identify the internal damage states of specimens in different periods, LDA classification model built by experiment of beech was utilized to training and testing the AE signals of Pinus sylvestris. The experimental results are shown in Fig. 13, and the classification evaluation indexes are shown in Table 6.

According to the classification results, the accuracy of the classification model reached $90.58 \%$. And, the model accurately divided the damaged AE signals of Pinus sylvestris into four types. That is, $0-50 \mathrm{~s}$ is the buckling $\mathrm{AE}$ signal, which the wood cell wall begins to buckle and collapse; $50-80 \mathrm{~s}$ is the deformation $\mathrm{AE}$ signal, which the wood cell wall interface begins to be damaged and delamination; $80-210 \mathrm{~s}$ is the micro-crack AE signal, which is the formation and expansion of the damage zone of wood micro-crack; $210-281 \mathrm{~s}$ is the fracture AE signal, which the wood cell wall fracture and continues to expand.

It can be seen that although implemented on different tree species, this method still achieves good classification accuracies. Among them, the classification accuracy of the Type 0 and Type 1 of AE signals is lower than others, which may be related to the smaller amount of data of these types of AE signals. However, this method can better distinguish the signals of Type 1 and Type 2 that are difficult to distinguish only based on the number of $\mathrm{AE}$ events. Last but not least, this method can accurately 


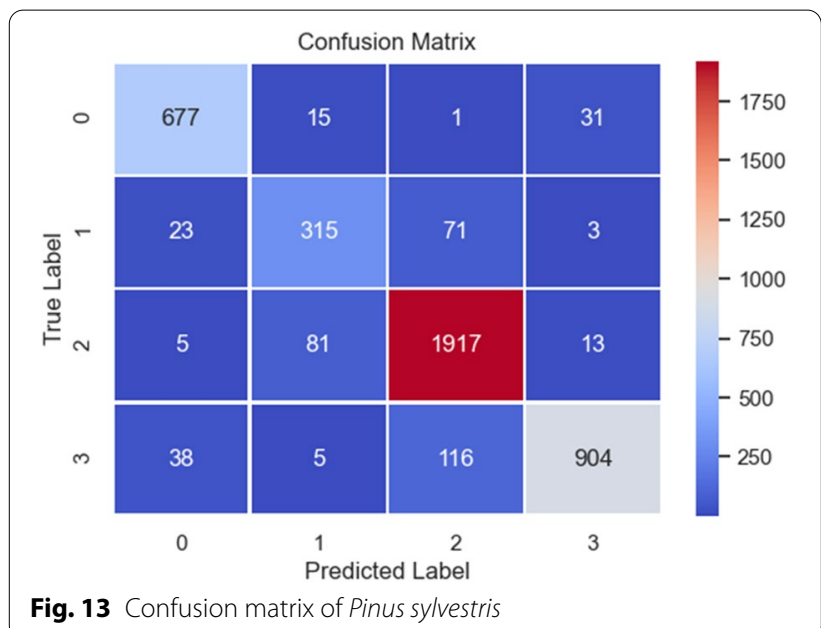

Table 6 Evaluation indicators

\begin{tabular}{lllll}
\hline \multicolumn{4}{l}{ Classification report } \\
\hline & Precision & Recall & F1-score & Support \\
\hline 0 & 0.91 & 0.94 & 0.92 & 724 \\
1 & 0.76 & 0.76 & 0.76 & 412 \\
2 & 0.91 & 0.95 & 0.93 & 2016 \\
3 & 0.95 & 0.85 & 0.90 & 1063 \\
Micro-avg & 0.91 & 0.90 & 0.90 & 4215 \\
Macro-avg & 0.88 & 0.88 & 0.88 & 4215 \\
Weighted avg & 0.91 & 0.90 & 0.90 & 4215 \\
\hline
\end{tabular}

classify the Type 2 and Type 3 that are difficult to distinguish based on the amplitude alone.

\section{Conclusion}

The nondestructive testing technology of AE signal for wood is of great significance for the evaluation of internal damages of wood. The research in this paper shows that: compared with typical statistical features, features introduced in this study such as entropy, crest factor, pulse factor, margin factor, and waveform factor have a higher sensitivity to damages; signal processing and feature extraction methods based on EMD and DWT can reduce computational costs and improve the accuracy of the classifier; the automatic classification model proposed in this paper has good $\mathrm{AE}$ signal recognition performance.

Moreover, this classification model lays a foundation for future research on the real-time monitoring and automatic identification of wood damage for wood material products such as building facilities, furniture materials, wooden ancient buildings, and music devices.

Future research includes: extending the model to different tree species; looking for more sensitive signal features, studying feature extraction and selection methods; adopting different machine learning classification algorithms; thereby establishing a higher accuracy and more generalized wood damage prediction model.

\section{Abbreviations}

AE: Acoustic emission; EMD: Empirical mode decomposition; DWT: Discrete wavelet transform; LDA: Linear discriminant analysis.

\section{Acknowledgements}

Not applicable.

\section{Authors' contributions}

ZM planned and implemented the research and wrote the manuscript. All the authors analyzed the results and approved the final manuscript. All authors read and approved the final manuscript.

\section{Funding}

Biological Resources Digital Development and Application Project of Yunnan Science and Technology Department (202002AA10007), Scientific Research Foundation of Yunnan Education Department (2020J0402, 2021 J0153).

\section{Availability of data and materials}

The datasets used and/or analyzed during the current study are available from the corresponding author on reasonable request.

\section{Declarations}

Competing interests

The authors declare that they have no competing interests.

Received: 15 June 2021 Accepted: 30 September 2021

Published online: 16 October 2021

\section{References}

1. Taghi T, Ying H (2001) Characterizing microscopic behavior of wood under transverse compression. Part II. Effect of species and loading djrection. Wood Fiber Sci 33(2):223-232

2. Girard L, Gruber S, Weber S, Beutel I (2013) Environmental controls of frost cracking revealed through in situ acoustic emission measurements in steep bedrock. Geophys Res Lett 40(9):1748-1753

3. Reiterer A, Stanzl-TscheggTschegg SEEK (2000) Mode I fracture and acoustic emission of softwood and hardwood. Wood Sci Technol 34(5):417-430

4. Choi NS, Woo SC, Rhee KY (2007) Effects of fiber orientation on the acoustic emission and fracture characteristics of composite laminates. J Mater Sci 42(4):1162-1168

5. Lamy F, Takarli M, Angellier N, Dubois F, Pop O (2015) Acoustic emission technique for fracture analysis in wood materials. Int J Fract 192(1):57-70

6. Fang SY, Qiu RZ, Li M (2018) Wood AE signal features based on improved EMD algorithm. J Vibrat Shock 37(23):292-298

7. Barile C, Casavola C, Pappalettera G, Vimalathithan PK (2019) Damage characterization in composite materials using acoustic emission signalbased and parameter-based data. Compos B Eng. https://doi.org/10. 1016/j.compositesb.2019.107469

8. Li XC, Li M, Ju S (2020) Frequency Domain Identification of Acoustic Emission Events of Wood Fracture and Variable Moisture Content. For Products J. 70(1):107-114

9. Jordan R, Feeney F, Nesbitt N, Evertsen JA (1998) Classification of wood species by neural network analysis of ultrasonic signals. Ultrasonics 36(1-5):219-222

10. Castellani M, Rowlands H (2009) Evolutionary artificial neural network design and training for wood veneer classification. Eng Appl Artif Intell 22(4-5):732-741 
11. Facciotto N, Martinez M, Troiani E (2017) Source identification and classification of acoustic emission signals by a SHAZAM-inspired Pattern Recognition Algorithm. Int Workshop Struct Health Monit. https://doi. org/10.12783/shm2017/13989

12. Tha B, Ying LA, Yy A, Qian ZA, Zh A (2020) Application of deep convolutional neural network on feature extraction and detection of wood defects. Sci Direct. Meas 152:107357

13. Wang W, Liu WD, Liu JM (2020) Acoustic Emission Recognition Based on Spectrogram and Acoustic Features. Artificial Intelligence and Security: 6th International Conference, ICAIS 2020 Hohhot, China, July 17-20, 2020 Proceedings, Part II. https://doi.org/10.1007/978-3-030-57881-7_66

14. Fathi H, Nasir V, Kazemirad S (2020) Prediction of the mechanical properties of wood using guided wave propagation and machine learning. Construct Building Mater 262:120848

15. Vs A, Jy A, Rk A, Dt B, Ae C, Pkm D (2020) On the rapid and non-destructive approach for wood identification using ATR-FTIR spectroscopy and chemometric methods. Vibrat Spectroscopy 110:103097

16. Rescalvo FJ, Suarez E, Valverde-Palacios I, Santiago-Zaragoza J, Gallego A (2018) Health monitoring of timber beams retrofitted with carbon fiber composites via the acoustic emission technique. Composite Struct 206:392-402

17. Rescalvo FJ, Morillas L, Valverde-Palacios I, Gallego A (2020) Acoustic emission in I-214 poplar wood under compressive loading. Eur J Wood Prod 78:723-732

18. Huang NE, Shen Z, Long SR, Wu MC, Shih HH, Zheng Q (1998) The empirical mode decomposition and the Hilbert spectrum for nonlinear and non-stationary time series analysis. Proc Math Phys Eng Sci 454(1971):903-995
19. Sun J, Xiao Q, Wen J, Wang F (2014) Natural gas pipeline small leakage feature extraction and recognition based on Imd envelope spectrum entropy and svm. Measurement 55(9):434-443

20. Peng C, Wang SS, He J, Li FJ (2021) Research on bearing fault diagnosis based on discrete wavelet transform and random forest. Appl Res Comput 1:101-105. https://doi.org/10.19734/j.issn.1001-3695.2019.09.0633

21. Karimian SF, Modarres M (2021) Acoustic emission signal clustering in cfrp laminates using a new feature set based on waveform analysis and information entropy analysis. Compos Struct 268:113987

22. MaT (2020) Analysis of sound signal of shearer experimental device based on LabVIEW. Anhui Univ Sci Technol. https://doi.org/10.26918/d. cnki.ghngc.2020.000052

23. Omar MF, Mansor MN, Saidi SA (2020) SFTA and GLCM via LDA Classifier for Skin Cancer Detection. IOP Conf Series Mater Sci Eng. https://doi.org/ 10.1088/1757-899X/932/1/012068

\section{Publisher's Note}

Springer Nature remains neutral with regard to jurisdictional claims in published maps and institutional affiliations.

\section{Submit your manuscript to a SpringerOpen ${ }^{\odot}$ journal and benefit from:}

- Convenient online submission

- Rigorous peer review

- Open access: articles freely available online

- High visibility within the field

- Retaining the copyright to your article

Submit your next manuscript at $\boldsymbol{\nabla}$ springeropen.com 